\title{
Unmasking Thyrotoxicosis in the Operating Room
}

Ana Martins Lopes ${ }^{1}$, Diana Leite ${ }^{1}$, Mariana Oliveira ${ }^{1}$, Rosa Maria Leite ${ }^{1}$

${ }^{1}$ Centro Hospitalar São João, E.P.E, Dept of Anaesthesiology, Porto, Portugal

\section{Background}

Thyrotoxicosis is a known cause of thyroid storm (TS) in patients exposed to triggers like surgery or infection.[1] TS is a rare life-threatening health condition, with a mortality rate of 10 to $30 \%$. The diagnosis is primarily clinical without specific signs or symptoms.

\section{Case Report}

\section{7 years-old female patient scheduled for esthetic breast reduction surgery}

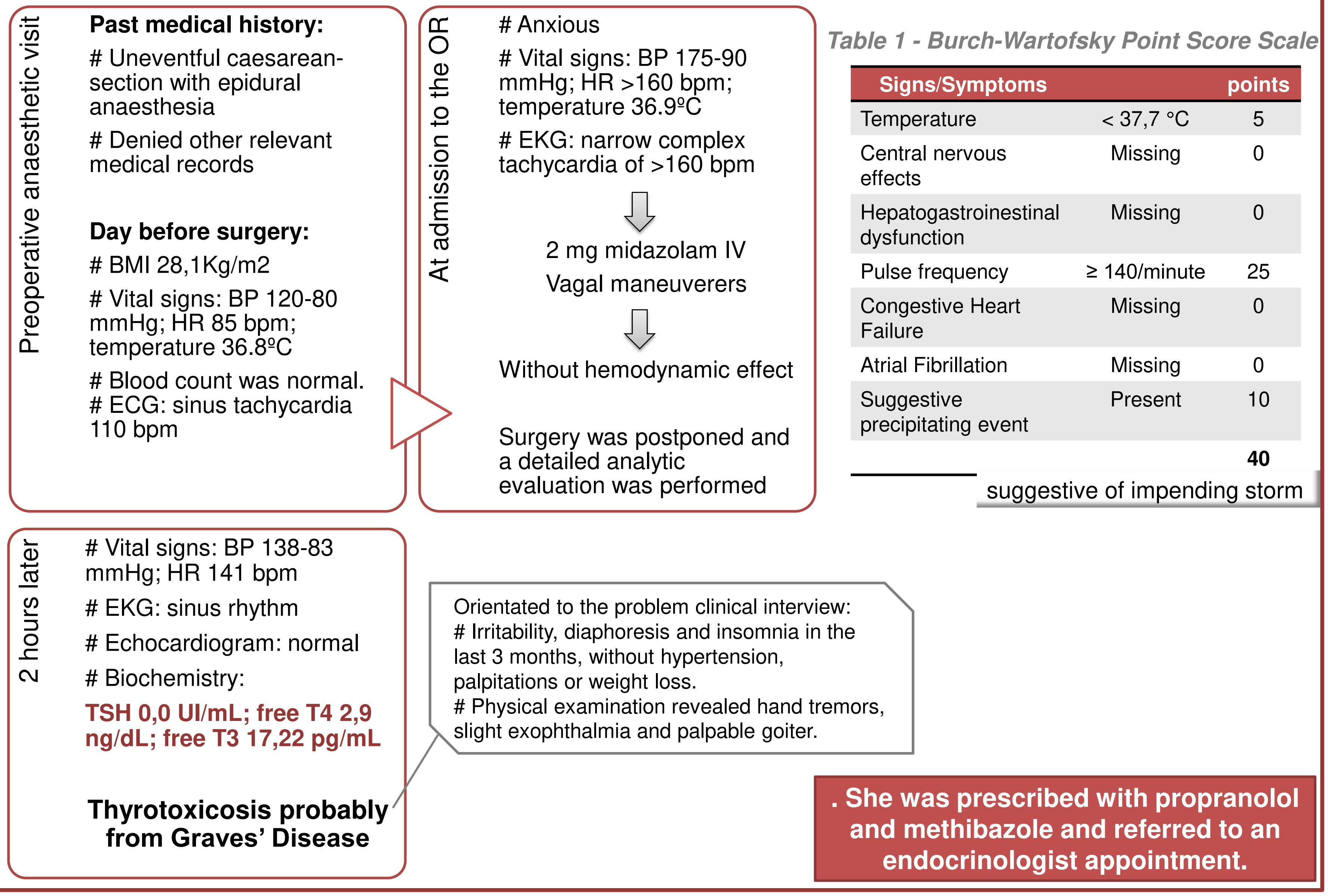

\section{Discussion}

$\checkmark$ Tachycardia and hypertension in a young patient, with overweight, can have a variety of diagnosis

$\checkmark$ Thyrotoxicosis is a rare event that demands the anaesthesiologist to be alert to its occurrence. Signs of thyroid storm include fever, rise in $\mathrm{PaCO} 2$, acidosis, hyperventilation, cardiovascular, neurologic or gastrointestinal dysfunction.

$\checkmark$ Treatment should be immediately started, as delays could be fatal.

\section{Learning points}

Thyroid pathology's symptoms can have a subtle beginning making diagnosis difficult, essentially when the phenotype is atypical. In the absence of a diagnose, having a high suspicious index can be the only way to, avoid serious complications in those patients. The anaesthesiologist should have an active role in the diagnosis and clinical management. 